\title{
A Differential Method for Representing Spinal MRI for Perceptual-CBIR
}

\author{
Marcelo Ponciano-Silva ${ }^{1,2}$, Pedro H. Bugatti ${ }^{3}$, Rafael M. Reis ${ }^{4}$, \\ Paulo M. Azevedo-Marques ${ }^{4}$, Marcello H. Nogueira-Barbosa ${ }^{4}$, \\ Caetano Traina-Jr. ${ }^{1}$, and Agma Juci Machado Traina ${ }^{1}$
}

1 Department of Computer Science, University of São Paulo at São Carlos, Brazil

2 Fed. Inst. of Education, Science and Technology of the Triângulo Mineiro, Brazil

3 Dept. of Computer Engineering, Fed. Tech. University of Paraná, Brazil

4 School of Medicine of University of São Paulo at Ribeirão Preto, Brazil

\{ponciano, pbugatti, agma, caetano\}@icmc.usp.br,

\{pmarques, rafael, marcello\}@fmrp.usp.br

\begin{abstract}
Image exams are a fundamental tool in health care for decision making. A challenge in Content-based image retrieval (CBIR) is to provide a timely answer that complies with the specialist's expectation. There are different systems with different techniques to CBIR in literature. However, even with so much research, there are still particular challenges to be overcame, such as the semantic gap. In this paper, we presented a new spinal-image comparison method based on the perception of specialists during his/her analysis of spine lesions. We take advantage of a color extractor and propose a shape descriptor considering the visual patterns that the radiologists use to recognize anomalies in images. The experiments shown that our approach achieved promising results, testifying that the automatic comparison of images should consider all relevant visual aspects and comparisons' criteria, which are defined by the specialists.
\end{abstract}

Keywords: Content-Based Medical Image Retrieval, Features Extraction, Spinal Images.

\section{Introduction}

X-Ray images, Magnetic Resonance Imagining (MRI) and Computed Tomography (CT) provide fundamental information for making an accurate diagnosis. In many situations, when a specialist gets a new image to analyze, having information and knowledge from similar cases can be very helpful. All these similar images are stored and organized by a system such as a Picture Archiving and Communication Systems (PACS). A PACS 7] consists of a set of computational systems able of providing storage, retrieval, distribution and presentation of images for physicians and medical staff in a Medical Center. One of its sub-systems allows specialists to have access to an automatic retrieval of similar cases based only on the intrinsic content of the image. These similar-cases retrieval subsystems use Content-Based Image Retrieval (CBIR) techniques [14].

J. Ruiz-Shulcloper and G. Sanniti di Baja (Eds.): CIARP 2013, Part I, LNCS 8258, pp. 464-471, 2013.

(C) Springer-Verlag Berlin Heidelberg 2013 
There are different systems that provide different techniques and algorithms to CBIR area of Spine. The Lister Hill National Center for Biomedical Communications department with the National Library of Medicine (NLM) is developing a CBIR for pathologies in the spine. This system supports hybrid queries, text-based and content-based of X-Ray images. The feature extraction is performed on Regions of Interest - ROIs (vertebral bodies), working with shape descriptors where each ROI is compared separately to each other [1]. There is also a tool developed for the Web, called SPIRS, which works on X-Ray images of the spine and makes a comparison between the separate ROIs [6]. Another study, focused on Spine MRI, uses statistical methods as Haralick features and texture spectrum spine MRI features for image feature extraction. This method is global, considering the entire image as the object for feature extraction. Thus, a single feature vector represents each image being used to compare it with another image contained in the base, in this case, to support diagnosis and treatment of scoliosis [9]. Another work allows automatically extracting specific measures from spine curvature of x-ray images from patients with scoliosis [10. In addition to these, there are also others generic systems, such as IRMA [5] and the ASSERT [12], among others, that can also incorporate specific techniques to the images discussed in this paper. CBIR tools have been extensively explored in several areas of medicine. However, even with so much research, there are particular challenges to be overcame in each specific area. The main one is the Semantic Gap [3], expressed as the difference between what the specialist considers as similar and what is offered as similar by the system. One of the techniques used to reduce this gap is to use of Perceptual-Parameters to guide the query according to well-defined user's Criteria of Similarity [11. However, to reach this level, there is the need to establish comparison methods compatible with the methods used by the radiologists in clinical practice and, therefore, to approximate the CBIR technique to the method identified.

In this paper we propose a spinal-image's comparison method based on the perception of the specialist during his/her analysis of lesions in the Lumbar Spine on MR images. To consolidate our proposal, we take advantage of a color extractor and propose a shape extractor to bring the CBIR to the way of the comparison performed by the physician during the process of analyzing images. The proposed approach achieved better results in the experiments, showing that the automatic comparison of images should consider all relevant visual aspects and criteria of comparison, defined by the specialist in a specific context.

\section{Related Concepts}

In the present section we show a set of significant visual patterns used as a similarity criterion for differentiating vertebral compression fractures - VCFs (focus of this work) as well as the traditional algorithms employed to extract the features to quantify the similarity between the images.

\subsection{Similarity Criterion for VCFs}

The practice of evidence-based medicine within radiology is growing and provides an important mechanism by which to facilitate further advancement of evidence 
based radiology. The goal of this practice is formalizing and standardizing image interpretation and results communication [15. Studying this interpretation allows to identify a set of significant visual predictors used by radiologists for differentiating VCFs and, with this knowledge to establish a criteria for comparing spinal images. Some diseases are characterized by the presence of known alterations in the normal vertebral body structure. The physicians call these alterations as radiological findings. Each radiological finding is related to visual feature parameters. For this work we listed some issues the radiologists analyze in spine images and for each issue it was specified what the radiologists look for in the image to help identifying a finding (see Figure 1).

\begin{tabular}{|c|c|c|c|}
\hline Finding & $\begin{array}{c}\text { Pathophysiological } \\
\text { Description }\end{array}$ & Visual Characteristics & Example \\
\hline \multirow{3}{*}{ 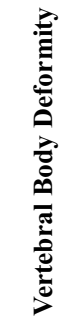 } & \multirow{3}{*}{$\begin{array}{l}\text { - Weakening of the } \\
\text { trabecular bone of the } \\
\text { vertebra and the } \\
\text { consequent } \\
\text { compression of the } \\
\text { vertebral body. } \\
\text { Trabecular bone is } \\
\text { impacting against } \\
\text { itself }\end{array}$} & $\begin{array}{l}\text { - Proportional Flattening: anterior } \\
\text { equivalent to posterior } \\
\text { - Decrease the Vertebra "height" }\end{array}$ & \\
\hline & & $\begin{array}{l}\text { - Flattening of Superior endplate } \\
\text { involvement. Change in shape }\end{array}$ & \\
\hline & & $\begin{array}{l}\text { - Flattening of Inferior endplate } \\
\text { involvement. Change in shape }\end{array}$ & \\
\hline \multirow{2}{*}{ 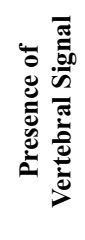 } & \multirow{2}{*}{$\begin{array}{l}\text { - Replacement of Fat } \\
\text { from Vertebral Body } \\
\text { by Edema }\end{array}$} & $\begin{array}{l}\text { - Change in Vertebral Color. } \\
\text { - Fat (normal) in MRI is bright, but } \\
\text { the edema is opaque (dark): } \\
\text { Distribution Homogeneous }\end{array}$ & \\
\hline & & $\begin{array}{l}\text { - Change in Vertebral Color. } \\
\text { - Fat (normal) in MRI is bright, but } \\
\text { the edema is opaque (dark): } \\
\text { Distribution Diffuse }\end{array}$ & \\
\hline
\end{tabular}

Fig. 1. Findings and visual characteristics

\subsection{Feature Extraction}

The feature vectors extracted from the medical images is the one of the key aspects for the similarity comparison between such images. To represent the similarity criterions showed in Subsection 2.1 we used the color and shape descriptors described as follows.

Color Descriptors. There are several methods to describe color features from images. The traditional gray-level histogram is one of the most applied one. However, some works have shown more robust alternatives to the color representation. One of this is an interesting feature extractor, called the Border/Interior Classification (BIC) 13. This color descriptor uses the RGB color space uniformly quantized in a given number of colors (e.g. each pixel represented by 6 bits). It presents a compact representation and consists in classifying the image pixels in edge-pixels or interior-pixels. Thus, it divides the image in two histograms. A pixel is classified as border if it is at the border of the image itself 
or if at least one of its 4-neighbors (top, bottom, left and right) has a different quantized color. A pixel is classified as interior if its 4-neighbors have the same quantized color. In the final step both histograms are merged, generating the image feature vector.

Shape Descriptors. Shape is one of the most important feature extracted from an image. For instance, in medical images shape-based features play a major role from describing the malignancy of tumor nodules to vertebral fractures. The Zernike moments [8] are a relevant shape-based features that employ the general theory of moments joined with the Zernike polynomials.

\section{Proposed Approach}

In this paper, we consider a search engine based on the radiologist's analysis as a Method Based on Human Perception (Perceptual-Based). The specialist's comparison method, when he/she is comparing two images, are not usually explored in the design of traditional CBIR systems. With the goal of bringing the CBIR system to the perception of the specialist, we questioned several radiologists about how they compare images that have the findings described in Subsection 2.1. After that, we developed ways to represent the visual aspects described for each finding. As a result of questioning, we got the proposals described as follows.

\subsection{Spine-Based Feature Extraction}

To represent the similarity criterions showed in Subsection 2.1 and aimed at employing the descriptors listed in Subsection 2.2, we propose the following color and shape descriptors.

Vertebral Body Color Descriptor. The BIC, presented in subsection 2.2 was designed for traditional RGB images. The medical images are usually in gray levels, with 16 bits per pixel. This format gives the images up to 4096 gray levels. Furthermore, the interior of objects found in medical images does not always have the same intensity (same pixel value). A slight variation in these gray levels within the same object can be considered normal in this context. Thus, using BIC can bring losses in traditional representation of the image, due to a possible misclassification between edge and interior.

Because of this observation, we have changed the way the pixel classification between interior/border, proposing the BIC-Med. With BIC-Med, the pixel is classified as border if at least one of its 4-neighbors (top, bottom, left and right) has a variation of quantized gray-level greater than a given threshold. A pixel is classified the interior if all its 4-neighbors have a variation less than or equal to a given threshold. In the final step both histograms are merged, generating the image feature vector. The threshold for the experiments of this work was $20 \%$ of the gray level of each pixel. This color variation value was chosen empirically based on an already established culture among physicians about the variation between the vertebrae images, as is presented below. 
Vertebral Body Shape Descriptor. The shape features extractor proposed in this work was created based on the method of analysis of vertebral bodies' fractures proposed by Genant et al. 4. The method is based on a given score from zero to three, considering the difference between the maximum and minimum measurement of the anterior $(\mathrm{AH})$, central $(\mathrm{CH})$ and posterior $(\mathrm{PH})$ vertebral body heights. It is assigned a score value of 0 (normal) to the percentage difference less than $20 \%$, until score 3 (severe fracture) to percentage difference greater than $40 \%$. Thus, the feature vector of the proposed extractor, called VertebralBody-RelativeShape (VB-RelativeShape), is composed of the relative proportion between the anterior, central and posterior vertebral body heights. That is, each vertebral body is represented by the proportion of three measurements. They are: $\mathrm{AH} / \mathrm{CH}, \mathrm{AH} / \mathrm{PH}, \mathrm{CH} / \mathrm{PH}$.

\subsection{Perceptual Spinal Based Method}

The images $A_{1}, A_{2}$ and $A_{3}$ of Figure 2 are quite similar. However, if you make a global comparison between them, will probably find images $A_{2}$ and $A_{3}$ more similar to each other. However, if you consider only the focusöf this specific domain (see $A_{1}^{\prime}, A_{2}^{\prime}$ and $A_{3}^{\prime}$ ), images $A_{1}$ and $A_{2}$ would be considered more similar to each other. This example characterizes the difference between what the specialist believes to be similar and that the system can deem to be similar (i.e., the semantic gap). Probably a descriptor of color or texture applied to the entire image (Global-Based) would erroneously return images $A_{2}$ and $A_{3}$ as the most similar ones.

On the other hand, in an attempt to reduce this semantic gap, many studies use ROIs for performing the comparison between images (ROIs-Based). However, although this method really brings good results, through graphs of precision and recall, it still has a gap in what occurs naturally during the expert analysis. The radiologist spots variations in ROIs contrasting them with neighboring regions: Not to mention that when comparing two images, 1) scoring ROIs is extra work to be performed by the radiologist and 2) When considering only similar ROIs, the

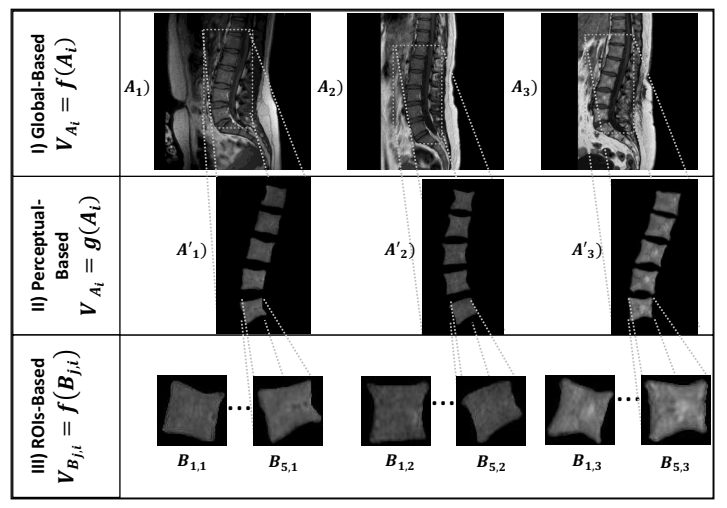

Fig. 2. Different methods (visions) for comparing images 
physician may come across images that have different ROIs representing (as is the case of Vertebral Bodies) having normal and non-normal ROIs in the same image.

In order to adapt the feature extraction for something close to the concept of similarity perceived by radiologists, we propose a method that considers the local characteristics (ROI-based), but, composed into a single feature vector. Thus, each vertebra belonging to the analyzed image is compared to the equivalent vertebra on another image. Thus, the similarity between two images is not only calculated by the similarity of a single ROI, but considering the variation of all the neighbors.

\section{Experiments and Results}

To evaluate the proposed method, we have generated precision and recall (P\&R) graphs 2]. Precision is the percentage of relevant images actually returned in the query among the total number of images returned in the query. Recall is the percentage of relevant images actually returned in the query among the total number of relevant images for a given query. For the dataset analyzed, all images of each class were employed as query centers.

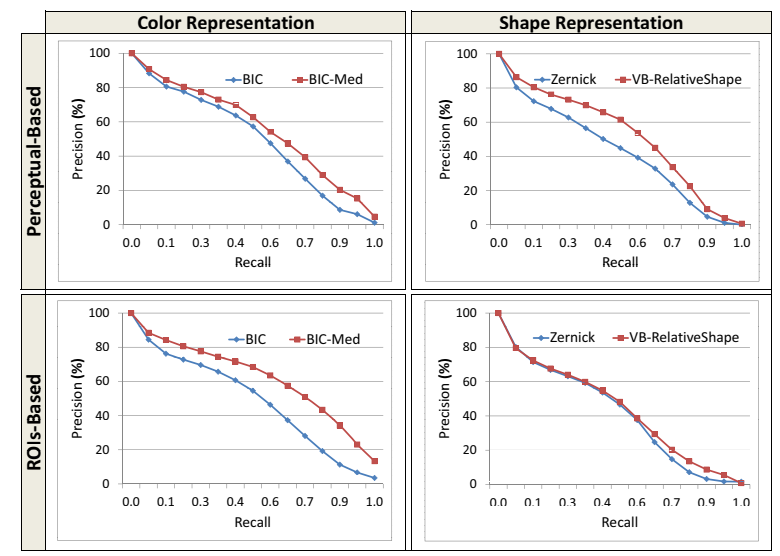

Fig. 3. Precision Vs. Recall graphs for Color and Shape representation

To perform the experiments we have used an image dataset, provided by the Hospital of our university (HCRP). The dataset consists of 171 images separated by the specialists into two distinct classes ( 83 normal images and 88 images with VCFs). To enable the Spinal-based experiments, radiologists and residents from HCRP provided a manual segmentation of the lumbar vertebral bodies. And to enable the ROI-based experiments, we used this manual segmentation to create a data set with 855 ROIs.

Analyzing the feature descriptor proposed in this work, both showed better performance when compared with traditional ones. This comparison was performed using data 171 images, through the Perceptual-based proposed method, 


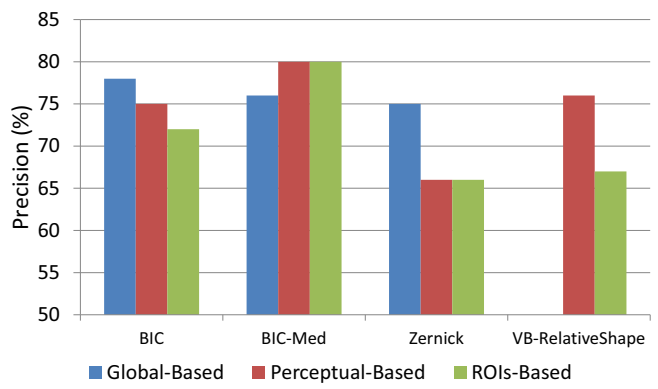

Fig. 4. Extractors' precision with different approaches considering the area under the Precision Vs.Recall graph in the first $50 \%$ of recall

and the base 855 ROIs (ROI-based) as shown in Figure 3 . Comparing the extractors color, the BIC-Med precision was higher than $80 \%$ in the first $20 \%$ of recall, while the traditional BIC has not reached this level. For shape extractors, considering the ROI-based methodology, both VB-RelativeShape and Zernike performed very similar. However, when analyzing with the focus on the Perceptual-based method, the precision of the two extractors improved and the VB-RelativeShape presented a significant gain compared to Zernike (about 10\% better in the first $20 \%$ of recall).

Another interesting result to be analyzed is the behavior of the different descriptors regarding different ways they are used to compare images (GlobalBased, Perceptual-based or ROI-based). Figure 4 illustrates their performance in the different approaches. The BIC and Zernike extractors showed are more focuses to analysis global image. They both lost performance when used in segmented images or ROIs. The BIC-Med extractor achieved better precision in almost all cases and was representative for Perceptual-Based and the ROIsbased retrieval. Already VB-RelativeShape showed much better precision with the proposed methodology, confirming the fact that specific features of shape, in this context, are best described as representing the whole neighborhood, not just a single ROI.

\section{Conclusions}

In this paper, we presented a new approach to deal with the semantic gap. We propose a spinal-image's comparison method based on the perception of specialist during his/her analysis of lesions in the Lumbar Spine on MR images. The proposed approach gathers from the physicians the visual patterns they use to recognize anomalies in images and apply them on building feature extractors based on these visual patterns. We took advantage of color extractor and propose a new shape extractor to bring the CBIR to the way of the comparison performed by the physician during the process of analyzing the images. The proposed approach achieved better results in the experiments, showing that the automatic comparison of images should consider all relevant visual aspects and criteria of comparison, defined by specialist in a specific context. 
Acknowledgments. We thank FAPESP, CAPES, CNPq and the INCT INCod for the financial support.

\section{References}

1. Antani, S., Lee, D.J., Long, L.R., Thoma, G.R.: Evaluation of shape similarity measurement methods for spine X-ray images. J. Visual Communication and Image Representation 15(3), 285-302 (2004)

2. Baeza-Yates, R.A., Ribeiro-Neto, B.A.: Modern Information Retrieval - the concepts and technology behind search, 2nd edn. Pearson Education Ltd., Harlow (2011)

3. Deserno, T.M., Antani, A., Long, L.R.: Ontology of Gaps in Content-Based Image Retrieval. J. Digital Imaging 22(2), 202-215 (2009)

4. Genant, H.K., Wu, C.Y., van Kuijk, C., Nevitt, M.C.: Vertebral fracture assessment using a semiquantitative technique. J. of Bone and Mineral Research: The Official J. of the American Society for Bone and Mineral Research 8(9), 1137-1148 (1993)

5. Güld, M.O., Thies, C., Fischer, B., Lehmann, T.M.: A generic concept for the implementation of medical image retrieval systems. I. J. Medical Informatics 76(23), 252-259 (2007)

6. Hsu, W., Antani, S., Long, L.R., Neve, L., Thoma, G.R.: SPIRS: A Web-based image retrieval system for large biomedical databases. I. J. Medical Informatics 78 (S1) S13-S24 (2009)

7. Huang, H.K.: PACS and Imaging Informatics: Basic Principles and Applications, 2nd edn. Wiley-Blackwell, Hoboken (2010)

8. Khotanzad, A., Hong, Y.H.: Invariant Image Recognition by Zernike Moments. IEEE Trans. Pattern Anal. Mach. Intell. 12(5), 489-497 (1990)

9. Kumaran, N., Bhavani, R.: Spine MRI Image Retrieval using Texture Features. I. J. of Computer Applications 46(24), 1-7 (2012)

10. Medina, J.M., Jaime-Castillo, S., Jiménez, E.: A DICOM viewer with flexible image retrieval to support diagnosis and treatment of scoliosis. Expert Syst. Appl. 39(10), 8799-8808 (2012)

11. Ponciano-Silva, M., Traina, A.J.M., Azevedo-Marques, P.M., Felipe, J.C., Traina, C.J.: Including the perceptual parameter to tune the retrieval ability of pulmonary cbir systems. In: CBMS, pp. 8-17 (2009)

12. Shyu, C.R., Brodley, C.E., Kak, A.C., Kosaka, A., Aisen, A.M., Broderick, L.S.: ASSERT: A Physician-in-the-Loop Content-Based Retrieval System for HRCT Image Databases. Comp. Vision and Image Understanding 75(1-2), 111-132 (1999)

13. Stehling, R.O., Nascimento, M.A., Falcão, A.X.: A compact and efficient image retrieval approach based on border/interior pixel classification. In: CIKM, pp. 102109 (2002)

14. Town, C.: Content-Based and Similarity-Based Querying for Broad-Usage Medical Image Retrieval. In: Sidhu, A.S., Dhillon, S.K. (eds.) Advances in Biomedical Infrastructure 2013. SCI, vol. 477, pp. 63-76. Springer, Heidelberg (2013)

15. Wang, K.C., Jeanmenne, A., Weber, G.M., Thawait, S.K., Carrino, J.A.: An Online Evidence-Based Decision Support System for Distinguishing Benign from Malignant Vertebral Compression Fractures by Magnetic Resonance Imaging Feature Analysis. J. Digital Imaging 24(3), 507-515 (2011)

16. Xue, Z., Long, L.R., Antani, S., Thoma, G.R.: Spine X-ray image retrieval using partial vertebral boundaries. In: CBMS, pp. 1-6 (2011) 